\title{
Eficacia de dos anestésicos tópicos, de uso dental, en pacientes pediátricos
} Maldonado-Ramírez MA ${ }^{1}$, Issasi-Hernández $\mathrm{H}^{1}$, Trejo-Tejeda $\mathrm{S}^{1}$, Morales-
Sánchez $\mathrm{LA}^{2}$

\section{Resumen}

INTRODUCCIÓN: el dolor a la punción durante la anestesia dental local puede disminuir con ayuda de un anestésico tópico, evitando asociar la anestesia dental con el dolor.

OBJETIVO: comparar la eficacia de dos anestésicos tópicos: a) lidocaína/prilocaína al 5\% (parche) y b) benzocaína al 20\% (gel) para disminuir la sensibilidad a la punción en la mucosa oral.

MATERIALES Y MÉTODOS: se diseñó un estudio clínico comparativo con 60 pacientes entre 3 y 9 años de edad, divididos en dos grupos experimentales; la punción se realizó con aguja dental calibre 30. La intensidad del dolor se evaluó mediante la escala SEM (sound, eyes, motor) contrastando los resultados con los cambios fisiológicos: saturación de oxígeno y pulso.

RESULTADOS: 60 niños de ambos géneros, de 3 a 9 años de edad, participaron en el estudio. La variable dolor-moderado se presentó en $15 \%$ de la población estudiada (9 participantes) de los cuales 6 fueron femeninas y 3 masculinos, todos pertenecían al grupo benzocaína. El análisis estadístico arrojó una mayor eficacia de lidocaína/ prilocaína ( $\mathrm{P}=0.025)$.

CONCLUSIONES: la aplicación de lidocaína/prilocaína al 5\% es más eficaz para reducir el dolor que produce la punción de la aguja de anestesia dental.

PALABRAS CLAVE: anestésicos tópicos, EMLA, benzocaína, dolor, anestesia dental.

Acta Pediatr Mex. 2017 Mar;38(2):83-90.

\section{Efficacy of two topical anesthetics for dental use in pediatric}

Maldonado-Ramírez $\mathrm{MA}^{1}$, Issasi-Hernández $\mathrm{H}^{1}$, Trejo-Tejeda $\mathrm{S}^{1}$, MoralesSánchez LA ${ }^{2}$

\section{Abstract}

BACKGROUND: Pain puncture during local dental anesthesia, can be reduced using a topical anesthetic, avoiding dental anesthesia associated with pain.
${ }^{1}$ Profesor de la Universidad Autónoma de Tamaulipas-Postgrado de Odontopediatría.

${ }^{2}$ Tesista del Postgrado de Odontopediatría de la Universidad Autónoma de Tamaulipas.

Recibido: 27 de marzo del 2016

Aceptado: 12 de septiembre del 2016

Correspondencia

Maldonado-Ramírez MA

odo.pediatrica.mario@gmail.com

mmaldona@uat.edu.mx

Este artículo debe citarse como

Maldonado-Ramírez MA, Issasi-Hernández $\mathrm{H}$, Trejo-Tejeda S, Morales-Sánchez LA. Eficacia de dos anestésicos tópicos, de uso dental, en pacientes pediátricos. Acta Pediatr Mex. 2017;38(2):83-90. DOI: http://dx.doi.org/10.18233/APM38No2pp83-901359 
OBJECTIVE: The aim of this study was to compare the efficiency of two oral topical anesthesics: a) Lidocaine/Prilocaine (patch) $5 \%$ vs b) Benzocaine (gel) $20 \%$, in paediatric patients.

DESIGN: Clinical comparative study. 60 patients divided into two experimental groups: a) and b), we apply a topical anesthesia to them, before realizing the puncture with dental needle caliber 30. Evaluation of the pain was based on the scale SEM (sound, eyes and motor), additionally we registered the oxygen saturation of and frequency of the heart rate.

RESULTS: 60 children of both genders from 3 to 9 years old participated in the study. The variable pain occurred in $15 \%$ of the study population (9 participants), of which 6 were female and 3 male. The statistical analysis showed a major efficiency of the patch. $(P=0.025)$.

CONCLUSIONS: The application of the patch was more effective in reducing the pain that produces the puncture of the needle of dental anesthesia.

KEYWORDS: topical anesthetics; EMLA; benzocaine; pain; dental anesthesia

\author{
${ }^{1}$ Profesor de la Universidad Autónoma de \\ Tamaulipas-Postgrado de Odontopediatría. \\ ${ }^{2}$ Tesista del Postgrado de Odontopediatría \\ de la Universidad Autónoma de Tamaulipas. \\ Correspondence \\ Maldonado-Ramírez MA \\ odo.pediatrica.mario@gmail.com \\ mmaldona@uat.edu.mx
}

\section{INTRODUCCIÓN}

La anestesia tópica es: "la supresión de la sensibilidad propioceptiva y dolorosa en cierta región del cuerpo, por la aplicación de un anestésico tópico en forma de solución, gel o ungüento sobre mucosas o piel intacta"; esta acción se debe al bloqueo, de forma reversible, de la conducción electroquímica que generan los impulsos nerviosos. ${ }^{1}$ Estudios previos mencionan que la aplicación tópica intraoral reduce la sensibilidad durante la punción de la aguja dental. ${ }^{2-6}$ De acuerdo con Malamed 7 la aplicación correcta del anestésico tópico permitirá la penetración de la aguja dental en la membrana de la mucosa oral de una manera indolora. Esta teoría ha sido apoyada por otros estudios en donde se comparó un agente anestésico con un placebo, dando como resultado que los agentes anestésicos utilizados fueron altamente eficaces y seguros al reducir el dolor a la punción; ${ }^{8-17} \sin$ embargo Kincheloe, en otro estudio, no encontró diferencias estadísticamente significativas. ${ }^{18} \mathrm{Al}$ respecto Martin y sus colaboradores sugieren que los anestésicos tópicos tienen más efecto psicológico que farmacológico. ${ }^{11}$

Algunos investigadores mencionan que existen factores que pueden afectar la eficacia de los anestésicos tópicos: a) zona anatómica; b) tiempo de aplicación y c) concentración del anestésico. ${ }^{15,17,19} \mathrm{~A}$ este respecto, algunos estudios afirman que la anestesia tópica reduce el dolor a la inserción de la aguja dental en la zona anterior superior. ${ }^{19,21}$ Hutchins y sus colegas ${ }^{22}$ concuerdan con esta teoría al publicar que la benzocaína al $20 \%$ redujo la incomodidad de la inyección en el lado bucal, pero su eficacia disminuye en el área palatina. Meechan y sus colegas ${ }^{16}$ concluyen que el grado de queratinización de las diferentes zonas anatómicas influye en la absorción del anestésico y en su acción. 
El tiempo de la aplicación es uno más de los factores que afectan la efectividad de los anestésicos tópicos. Al aumentar el tiempo de exposición a la anestesia tópica aumenta su efectividad; así, publicaciones como las de Rosivack y su grupo ${ }^{6}$ que utilizan un tiempo de aplicación de tres minutos para la benzocaína de forma efectiva. Svensson y sus colaboradores ${ }^{8}$ informaron que una aplicación de cinco minutos de EMLA (lidocaína/prilocaína al 5\%) en el paladar reducía más el dolor de las inyecciones a comparación del placebo. También Hutchins y sus colegas ${ }^{21}$ reportaron el éxito de la aplicación de un minuto de benzocaína en comparación con el placebo. Años después Al-Melh y otros ${ }^{5}$ informaron que una aplicación de lidocaína/prilocaína al 2.5\% y la crema EMLA son de mayor efectividad para reducir el dolor cuando se aplican por diez minutos. Otro aspecto a considerar es la concentración del anestésico y Hersh y sus colegas $^{9}$ comentan que a mayor concentración del fármaco, mayor eficacia para inhibir el dolor durante la inserción de la aguja dental; Tatay y su grupo ${ }^{1}$ mencionan que el tiempo de aplicación está en relación con la concentración del anestésico. Con todo, Primosch y su equipo ${ }^{17}$ no recomiendan el uso de EMLA en niños por su mayor tiempo de aplicación en comparación con la benzocaína al $20 \%$. Sin embargo, Wu y sus colaboradores ${ }^{22}$ estudiaron la preferencia en los pacientes y aseguran que los niños prefirieron el uso del parche de lidocaína en comparación con el gel. Otro factor a tomar en cuenta es la edad y género de los sujetos a estudiar, al respecto Ruda, Ramírez y Palmeira afirman que aunque no existen estudios concluyentes parece haber diferencias en la percepción del dolor y la respuesta a dicho estímulo entre el género y la edad. ${ }^{23-25}$

Hoy en día, el control del dolor provocado por la inyección del anestésico local en pacientes pediátricos continúa siendo la piedra angular para lograr la cooperación y adecuada conducta del paciente durante el tratamiento dental y esto motiva el rechazo de las visitas al consultorio dental por parte de los pacientes mismos y de sus padres. Lo anterior justifica un estudio con el objetivo de comparar la efectividad de dos anestésicos tópicos usados en pacientes pediátricos. Actualmente contamos sólo con dos productos de uso tópico para aplicar en la cavidad oral como son la benzocaína en presentación de gel o spray y la lidocaína en presentación de crema o spray, pero en el mercado existe un producto con la presentación en forma de parche que contiene una combinación de lidocaína/prilocaína, el cual pudiera presentar ventajas sobre los ya conocidos. Nuestra hipótesis de estudio es que existe diferencia en la eficacia entre el gel de benzocaína al $20 \%$ y el parche de lidocaína/ prilocaína al 5\%.

\section{OBJETIVO}

Comparar la eficacia de dos anestésicos tópicos en el control del dolor a la punción ocasionado por la aguja dental, durante la infiltración de la anestesia local en la mucosa vestibular maxilar en la región de incisivos en pacientes pediátricos. La hipótesis de estudio es que existe diferencia en la efectividad anestésica entre la benzocaína y la lidocaína/prilocaína.

\section{MATERIALES Y MÉTODOS}

Se diseñó un estudio clínico comparativo para identificar el anestésico tópico de mayor eficacia. El consentimiento informado se obtuvo de los padres o tutores de los pacientes, así como la aceptación verbal del paciente pediátrico.

La muestra fue de 60 pacientes sanos, entre los 3 y 9 años de edad, de ambos géneros, que acudieron al Servicio de Odontopediatría con necesidad de tratamiento operatorio, sin antecedentes previos. Los niños seleccionados tenían una encía sana, sin historia de alergias a los 
componentes de los anestésicos. Se excluyeron a aquellos pacientes que se negaron a participar en el estudio, que tuvieran alteraciones de la encía en la zona seleccionada para la punción o enfermedad sistémica. No se eliminó a ninguno de las participantes ya que no se presentaron efectos adversos durante el estudio.

Los niños seleccionados fueron divididos en 2 grupos de 30 niños cada uno: grupo A (EMLA) y grupo B (benzocaína). Con la finalidad de observar si la edad y género poseen influencia en los resultados, cada grupo a su vez se subdividió en cuatro grupos, dando un total de ocho grupos; la subdivisión fue de acuerdo a la edad ( 3 a 6 años y 7 a 9 años) y de acuerdo al género (masculino y femenino).

La asignación de grupo A o B de los participantes fue mediante método no probabilístico, sin ocultación del mismo, a los pacientes se les proporcionó un número del 1 al 60 conforme fueron aceptados para el estudio, los números nones se asignaron al grupo A y los números pares al grupo B. El tamaño de la muestra se determinó de acuerdo al número de pacientes que solicitaron el servicio (por conveniencia) incluyendo a $100 \%$ de los pacientes que necesitaron tratamiento operatorio y presentaron los criterios de inclusión en un periodo de dos meses.

A cada grupo se le asignó a uno de los dos agentes de prueba de la siguiente manera: grupo A: lidocaína/prilocaína al 5\% (EMLA, AstraZeneca) y grupo B: benzocaína al 20\% (topicaína Zeyco).

La zona anatómica seleccionada para puncionar fue la mucosa vestibular alveolar del incisivo superior derecho, debido a que esta proporciona la facilidad de acceso y aislamiento necesarios. Previo a la aplicación la zona se desinfecto con algodón embebido en solución de iodopolivinilpirrolidona (Isodine ${ }^{\mathrm{MR}}$ ). Después de realizar la asepsia y del aislamiento relativo con rollos de algodón estéril, el área de prueba se secó usando una gasa estéril; con una cucharilla de medidas (GC Gold Label) se tomaron $2.7 \mathrm{~g}$ del anestésico, de los cuales sólo se empleó la mitad del contenido desechándose el restante y con ayuda de un aplicador de algodón se colocó en la zona anatómica seleccionada.

Para poder obtener un valor de referencia, en la sala de espera y en presencia de sus padres, antes de introducir el paciente al área clínica, se registraron la saturación de oxígeno y la frecuencia del pulso.

La aplicación de los anestésicos tópicos se realizó en el sillón dental de forma convencional de acuerdo a los informes de Svensson, Hersh, Primocsh, Carr y Wu. ${ }^{8-10,17,22} \mathrm{El}$ anestésico en contacto con la mucosa se dejó por 5 minutos (EMLA) y 3 minutos (benzocaína), la colocación del anestésico tópico la realizó un estudiante de segundo año del postgrado de odontopediatría, y pasado el tiempo de aplicación, se realizó la punción dando tiempo suficiente a los evaluadores para registrar los cambios faciales, comportamiento del paciente, saturación de oxígeno y frecuencia de pulso. Para el registro de los datos se asignaron dos estudiantes que se encargaron de llenar el formato de captura de datos con ayuda de oxímetro de pulso (Fingertip pulse oximeterMod. MD300C54) y la escala: sonidos, ojos, movimiento motor (SEM por sus siglas en inglés). Los estudiantes fueron previamente entrenados para la evaluación y registro logrando un índice Kappa de 80.

Tanto el operador como los evaluadores siempre fueron los mismos para evitar el sesgo de variabilidad en la habilidad de la punción y de interpretación de las escalas de medición sin cegamiento del operador ni de los evaluadores.

La variable dolor se evaluó de maneras subjetiva y objetiva: para la metodología subjetiva se 
utilizó la escala del dolor SEM (Sound, Eyes, Motor) utilizada por Wright ${ }^{26}$ y con metodología objetiva registraron: saturación de oxígeno $\left(\mathrm{SpO}_{2}\right)$ y frecuencia del pulso (bpm) con ayuda de oxímetro digital (Fingertip Pulse Oximeter Modelo: MD300C54). Los datos fueron asegurados y analizados con ayuda del programa Excell $^{\mathrm{MR}}$. Para la estadística descriptiva se utilizaron tablas de frecuencia y para la estadística inferencial se utilizó la prueba $\mathrm{Chi}^{2}$ para la variable dolor, para la variable saturación de oxígeno utilizamos la prueba no paramétrica de U-Mann Whitney (ya que la variable no posee una distribución normal) y para la variable frecuencia de pulso usamos la prueba paramétrica para comparación de medias con distribución normal T-student.

\section{RESULTADOS}

El total de participantes fue 60 y todos finalizaron el estudio, se dividieron en dos grupos de 30 participantes cada uno, de ambos géneros, y se formaron 8 subgrupos por edad (3-6 años y 7-9 años), la distribución de frecuencias (participantes) en cada grupo y subgrupos se muestra en el Cuadro 1.

Las características basales previas al estudio de los participantes se encontraron dentro de los valores normales para su edad y género, la

Cuadro 1. Distribución de frecuencias por grupo (subgrupo), género y edad

\begin{tabular}{|l|c|c|c|}
\hline Grupo (Subgrupo) & Género & Edad & Total \\
\hline Gel (1) & F & 3-6 años & 8 \\
\hline Gel (2) & F & 7-9 años & 7 \\
\hline Gel (3) & M & 3-6 años & 7 \\
\hline Gel (4) & M & 7-9 años & 8 \\
\hline Parche (5) & F & 3-6 años & 7 \\
\hline Parche (6) & F & 7-9 años & 8 \\
\hline Parche (7) & M & 3-6 años & 7 \\
\hline Parche (8) & M & 7-9 años & 8 \\
\hline
\end{tabular}

saturación de oxígeno observada fue de $97 \%$ y el pulso fue de 92 en promedio.

Del total de niños que participaron en el estudio sólo 15\% (9 participantes) mostro algún signo de dolor; en 6 de ellos se registró dolor moderado y en 3 dolor leve, ningún participante registro dolor intenso. En cuanto a la proporción observada en la frecuencia por edad y género e intensidad del dolor, dos participantes femeninas en el rango de 3 y 6 años de edad mostraron dolor moderado, mientras que en el mismo rango de edad, pero del género masculino, sólo uno registró dolor moderado y otro dolor leve. En el rango de 7 a 9 años de edad dos participantes femeninas registraron dolor moderado y dos más dolor leve, mientras que en el género masculino sólo uno registró dolor leve. En total, la distribución por género de los participantes que registraron dolor fue de 6 participantes femeninas contra 3 participantes masculinos, la distribución por rango de edad fue de cuatro para el rango de 3 a 6 años y cinco para el rango de 7 a 9 años. La proporción por edad y género fue mayor para participantes femeninas y para el rango de 7 a 9 años de edad, siendo mayor la diferencia por género (el doble) que por rango de edad.

El Cuadro 2 muestra la variación del pulso (método objetivo) evidenciando mayor eficacia del anestésico tópico usado en el grupo B. La prueba T-student de diferencia de medias confirma la diferencia estadísticamente significativa a favor del EMLA ( $P=0.025)$ (Cuadro 3).

El Cuadro 4 exhibe el porcentaje de eficacia con el método de medición subjetiva (escala SEM). El grupo EMLA registró 2 participantes que percibieron dolor leve, mientras que el grupo benzocaína registro 20 participantes que percibieron dolor, 11 de ellos en grado leve y 9 en grado moderado. La mayor eficacia se presentó en el grupo B (93\% de éxito) en comparación con el grupo A (60\% de éxito). Estos datos con- 
Cuadro 2. Valores promedio del pulso por grupo y porcentajes de variación del pulso

\begin{tabular}{|c|c|c|c|c|c|c|}
\hline \multicolumn{2}{|c|}{ Grupo } & \multirow{2}{*}{$\begin{array}{c}\text { MEDIA } \\
\text { Pulso inicio } \\
87\end{array}$} & \multirow{2}{*}{$\begin{array}{c}\text { MEDIA } \\
\text { Pulso punción } \\
101.14\end{array}$} & \multirow{2}{*}{$\begin{array}{c}\text { MEDIA } \\
\text { Pulso post } \\
103.28\end{array}$} & \multirow{2}{*}{$\begin{array}{c}\text { \% aumentó } \\
18.77 \%\end{array}$} & \multirow{2}{*}{$\begin{array}{c}\text { Pulsaciones/min } \\
\qquad 16.28\end{array}$} \\
\hline 1 & Gel Fem $3-6^{*}$ & & & & & \\
\hline 2 & Gel Fem $7-9^{*}$ & 93.44 & 98.77 & 96.11 & $2.85 \%$ & 2.67 \\
\hline 3 & Gel Masc $3-6^{*}$ & 90.14 & 90.85 & 93 & $3.17 \%$ & 2.86 \\
\hline \multirow[t]{2}{*}{4} & Gel Masc 7 - 9* & 87.25 & 96.62 & 92.25 & $5.73 \%$ & 5 \\
\hline & & & & & \% disminuyó & pulsaciones/min \\
\hline 5 & Parche Fem $3-6^{*}$ & 95.87 & 88.75 & 93.5 & $2.47 \%$ & 2.37 \\
\hline 6 & Parche Fem $7-9^{*}$ & 98.62 & 90.75 & 84.25 & $14.57 \%$ & 14.37 \\
\hline 7 & Parche Masc 3- 6* & 97.14 & 97.28 & 89.71 & $7.64 \%$ & 7.43 \\
\hline 8 & Parche Masc 7-9* & 89.12 & 88.12 & 84.87 & $4.76 \%$ & 4.25 \\
\hline
\end{tabular}

*Edad en años.

Cuadro 3. Resultado de la prueba estadística $t$ student

\begin{tabular}{|l|c|c|c|}
\hline $\begin{array}{l}\text { Media } \\
\text { pulso }\end{array}$ & $\begin{array}{c}\text { Pulso } \\
\text { inicio }\end{array}$ & $\begin{array}{c}\text { Pulso } \\
\text { punción }\end{array}$ & $\begin{array}{c}\text { Pulso } \\
\text { posterior }\end{array}$ \\
\hline EMLA & 94.733 & 91.1 & 87.63 \\
\hline GEL & 88.80 & 97.46 & 95.50 \\
\hline P-value & $\mathrm{P}=(0.095)$ & $\mathrm{P}=(0.137)$ & $\mathrm{P}=(0.025)$ \\
\hline
\end{tabular}

Cuadro 4. Resultados de la medición subjetiva de dolor, escala SEM de 60 pacientes

\begin{tabular}{|c|c|c|c|}
\hline & Leve & Moderado & Sin dolor \\
\hline \multicolumn{4}{|c|}{ Grupo- Dolor Sonido } \\
\hline EMLA & $1(3.33 \%)$ & $0(0 \%)$ & $29(96.66 \%)$ \\
\hline GEL & $2(6.66 \%)$ & $6(20 \%)$ & $22(73.33 \%)$ \\
\hline \multicolumn{4}{|c|}{$\mathrm{P}=(0.026)$} \\
\hline \multicolumn{4}{|c|}{ Grupo- Dolor $\underline{\text { Ojos }}$} \\
\hline EMLA & $2(6.66 \%)$ & $0(0 \%)$ & $28(93.33 \%)$ \\
\hline GEL & $11(36.66 \%)$ & $1(3.33 \%)$ & $18(60 \%)$ \\
\hline \multicolumn{4}{|c|}{$\mathrm{P}=(0.009)$} \\
\hline \multicolumn{4}{|c|}{ Grupo- Dolor Motor } \\
\hline EMLA & $2(6.66 \%)$ & $0(0 \%)$ & $28(93.33 \%)$ \\
\hline GEL & $8(26.66 \%)$ & $3(10 \%)$ & $19(63.33 \%)$ \\
\hline $\mathrm{P}=(0.0$ & & & \\
\hline
\end{tabular}

firman la hipótesis al encontrar una diferencia estadísticamente significativa en favor del EMLA.
A pesar de la variación registrada en las frecuencias de pulsaciones por minuto, no se presentaron diferencias entre grupos en los niveles de saturación de oxígeno (Cuadro 5).

\section{DISCUSIÓN}

Las variaciones en los estudios publicados hacen muy difícil comparar los resultados debido a que cada autor utiliza un material y método diferentes, pero la información recabada en ellos sirvió para fundamentar nuestro estudio de manera que pudiéramos comparar dos anestésicos de mayor aceptación en odontopediatría, con las dos técnicas utilizadas más frecuentemente en la evaluación del dolor.

Después de analizar las publicaciones encontradas se decidió obtener un promedio de los tiempos para la aplicación de cada anestésico

Cuadro 5. Saturación de oxígeno por grupo: antes, durante y posterior

\begin{tabular}{lc|c|c|c|}
\hline MEDIA SpO & $\mathrm{SpO}_{2}$ inicio & $\mathrm{SpO}_{2}$ punción & $\mathrm{SpO}_{2}$ posterior \\
\hline EMLA & 97.90 & 97.46 & 97.33 \\
Gel & 97.86 & 97.03 & 97 \\
\hline P-Value & $\mathrm{P}=(0.865)$ & $\mathrm{P}=(0.773)$ & $\mathrm{P}=(0.947)$
\end{tabular}


tópico: cinco minutos para el EMLA y tres minutos para la benzocaína, esta diferencia se debe a la mayor disponibilidad de la benzocaína para su absorción y la zona anatómica elegida fue la recomendada por los diferentes autores (mucosa no queratinizada), siendo la mucosa alveolar del maxilar superior del área de incisivos la seleccionada.

Al evaluar los resultados arrojados en la medición objetiva se hace evidente la reducción en la frecuencia del pulso, este dato se puede interpretar como un estado de relajación del paciente debido a que se encuentra confortable en el momento de la lectura de este signo; al no percibir la punción su estado de alerta se mantiene estable y al permanecer inmóvil pasa a un estado de relajación mayor, lo que hace posible que su frecuencia de pulso baje. Cabe destacar que el hecho de no tener experiencias previas al procedimiento hace posible mantener al paciente tranquilo y cooperador, bajo estas condiciones funcionan mejor las técnicas de manejo de conducta dentro del consultorio dental, esto se pudo comprobar al aplicar la prueba estadística de diferencias de medias para grupos independientes (prueba $T$ student), dando una diferencia estadísticamente significativa $(p=0.025)$ en favor del anestésico tópico EMLA. Los datos que se muestran en el Cuadro 3 pueden evidenciar que en el grupo de la benzocaína sí hubo una alteración con un ligero aumento en la frecuencia del pulso, lo que interpretamos como una percepción de la punción alcanzando cierta incomodidad del paciente (sin ocasionar su falta de cooperación); el estado de alerta y realizar pequeños movimientos en sus caras o extremidades explica el aumento del pulso que se registró. Estos resultados coinciden con los de Rosivack, Nayak, Al-Melh, Valle y Leyda ${ }^{1-6}$ donde la aplicación de anestesia tópica proporcionó disminución de la sensibilidad a la punción causada por la aguja dental.
Con estos resultados diferimos de las afirmaciones de Kincheloe y Martin ${ }^{11,18}$ ya que al utilizar dos métodos distintos para medir el dolor; comprobamos que además del comportamiento (escala SEM subjetiva-efecto psicológico) también ocurren cambios a nivel fisiológico (medición objetiva) al variar el pulso con la sensación experimentada a la punción de la aguja dental.

Sin embargo, este estudio sólo incluyó a pacientes de primera vez, sin experiencias previas de atención dental, lo que impide tener grupos con un número mayor de participantes. Creemos importante realizar más estudios en los que se incluya a pacientes con experiencias previas y aumentar el número de sujetos para estar completamente seguros de la eficacia encontrada. También, en un intento por mejorar el estudio, de ser posible habría que cegar tanto al operador como a los evaluadores. Estos resultados pueden extrapolarse inicialmente para atender a pacientes de primera vez con la seguridad de que estarán más confortables durante su tratamiento al utilizar éste método.

\section{CONCLUSIONES}

1. La aplicación del parche con lidocaína/prilocaína al 5\% (EMLA) posee mayor eficacia para controlar el dolor causado por la punción de la aguja dental.

2. Con la aplicación del parche con lidocaína/ prilocaína al 5\% (EMLA) el pulso del paciente disminuye aún después de aplicar la punción de la aguja dental.

\section{REFERENCIAS}

1. Tatay VJ, Anestésicos tópicos: revisión. Rev. Esp. Anestesiol. Reanim. 2009;56(2):S1-9.

2. Valle C, Ruiz D, Diez C. Eficacia del uso odontológico de la anestesia tópica previa a la punción anestésica infiltrativa. Estudio doble ciego. Avances en Odontoestomatología. 2011;(27)99-105. 
3. Leyda A., Llena C. Comparation of the eutectic mixture of lidocaine/prilocain versus benzocaine gel in children. Journal of Stomatology. 2011;(1):84-91.

4. Nayak R, Sudha P, Evaluation of three topical anaesthetic agents against pain: A clinical study. Indian J Dent Res. 2006;(17):155-160.

5. Al-Melh MA, Andersson L. Comparison of topical anesthetics (EMLA / Oraqixvs benzocaine) on pain experienced during palatal needle injection. Oral Surg Oral Med Oral Pathol Oral RadiolEndod. 2007;103:e16-e20.

6. Rosivack RG, Koenigsberg SR, Maxwell KC. An analysis of the effectiveness of two topical anesthetics. AnesthProg. 1990;37(6):290-292.

7. Malamed, S. Handbook of local anesthesia, 4th Ed. Mosby. 1997. pp. 51-58.

8. Svensson P, Petersen JK: Anesthetic effect of EMLA occluded with orahesive oral bandages on oral mucosa. A placebo-controlled study. Anesth Prog. 1992;39:79-82.

9. Hersh E, Houpt M, Cooper S, Feldman R, Wolff M, Levin L. Analgesic Efficacy and safety of an intraoral lidocaine patch, J Am Dent Assoc. 1996;(127):1626-1634.

10. Carr M, Horton J, Evaluation of a transoral delivery system for topical anesthesia. J Am Dent Assoc. 2001;(132):1714-1719.

11. Martin MD, Ramsey DS, Whitney C, Fiset L, Weinstein P. Topical anesthesia: differentiating the pharmacological and psychological contributions to efficacy. AnesthProg. 1994;41:40-47.

12. Grover K., Samadi F., Jaiswal J.N., Navit S., Saha S. Local Anaesthetic Agents In Pediatric Dentistry. J of Int. Dental and Medical Research. 2012;5(2):96-101.

13. Mayor-Subirana G., Yagüe-García J., Valmaseda-Castellón E., Arnabat-Domínguez J., Berini-Aytés L., Gay-Escoda C. Anesthetic efficacy of oraqix ${ }^{\circledR}$ and placebo for pain control during non-surgical periodontal treatment. Med Oral Patol Oral Cir Bucal. 2014;1;19(2):192-201.

14. Varma S., Bhola S., Mohitey J., Redasani R., Gangavati R., Koregol A. Effectiveness of a novel topical anesthetic gel in patients undergoing non surgical periodontal therapy. CumhuriyetDent J. 2013;16(2):99-103.

15. Meechan J. Intraoral topical anesthesia. Periodontology 2000. 2008;46:56-79

16. Bagesun M., Tabrizi P. Lidocaine $20 \%$ patch vslicocaine $5 \%$ gel for topical anaesthesia of oral mucosa. Int. J. of Paed Dent. 2008;18:452-460.

17. Primosch R, Rolland G, Comparison of topical EMLA 5\% oral adhesive to benzocaine $20 \%$ on the pain experienced during palatal anesthetic infiltration in children. Pediatric Dentistry. 2000;(23)11-14.

18. Kincheloe JE, Mealiea WL, Jr, Mattison GD, Seib K Psychophysical measurement on pain perception after administration of a topical anesthetic. Quintessence Int. 1991;22(4):311-315.

19. Nusstein J, Beck M. Effectiveness of $20 \%$ Benzocaine as a topical anesthetic for intraoral injections. AnesthProg. 2003;(50):159-163.

20. Nakanishi O, Haas D, Ishikawa T, Kameyama S, Nishi M. Efficacy of mandibular topical anesthesia varies with the site of administration. AnesthProg. 1996;43:14-19.

21. Hutchins HS, Young FA, Lackland DL, Fishburne CP. The effectiveness of topical anesthesia and vibration in alleviating the pain of oral injections. AnesthProg 1997:44:87-89.

22. Wu SJ, Julliard K. Children's Preference of Benzocaine Gel Versus the Lidocaine Patch. Pediatr Dent. 2003;(25):401-405.

23. Ruda MA. Gender and Pain. Pain. 1993;63:1-2.

24. Ramírez M.C, Esteve R, LópezM. AE and Anarte Mạ T. Differences in the perception of pain related to sex and age variables. Rev SocEsp Dolor. 2001;8:562-568.

25. Palmeira CCA, Ashmawi HA, Posso IP - Sexo y Percepcióndel Dolor y Analgesia.Sexo y Percepcióndel Dolor y Analgesia. Rev Bras Anestesiol. 2011;61(6):449-458.

26. Wright GZ, Weinberger SJ, Marti R, Plotzke O. The effectiveness of infiltration anesthesia in the mandibular primary molar region. Pediatr Dent. 1991;13:278-82.a 